\title{
Closing the Loop
}

\author{
Eyal Dassau, ${ }^{1,2}$, Thomas Hennings ${ }^{2}$, Jane Fazio ${ }^{2}$, Eran Atlas ${ }^{3}$, and Moshe Phillip ${ }^{3,4}$
}

\section{Introduction}

$\mathbf{F}$ OR MANY PATIENTS WITH DIABETES, maintaining good glycemic control can be a full-time job, and when this extra job is added into people's busy lives, even the most dedicated and motivated patients can have trouble keeping up. Because of the tremendous burden that diabetes places on society and on the individual, much attention has been paid to the development of closed-loop artificial pancreas (AP) systems capable of automated blood glucose (BG) management. In contrast to conventional blood glucose management therapies, a perfect closed-loop system will be able to measure glucose and correspondingly administer insulin to maintain BG levels within a specified range. In addition, it will be able to compensate for other factors that can make BG management much more complicated, like exercise, illness, stress, and temporal changes in insulin sensitivity. Progress is being made, slowly and surely, and will continue in small steps. We have already seen the implementation of nocturnal closedloop control in Europe. From there, the next step may be daytime automation of basal rates with or without userdetermined pre-meal boluses, followed perhaps by full automation and bihormonal systems. Even transferring a small amount of responsibility from the patient to the system, as in nocturnal closed-loop control, can have a huge impact on quality of life. The path to a commercial fully closed-loop artificial pancreas system might still pose a challenge, but there is a growing diversity of ideas and interest in the field.

The past year appears to have been one of transition. The small number of clinical papers published does not indicate a lack of interest. Rather, it is more likely that the results of the clinical trials undertaken during this period are not yet ready for publication. The use of an AP for perioperative glucose management is still debatable. Its use in the ICU is an example of additional functions for closed-loop control outside of diabetes. We have summarized here articles that have demonstrated significant advancements in the closed-loop AP field and that have the potential to make the greatest impact.

\section{ARTIFICIAL PANCREAS FOR TYPE 1 DIABETES}

\section{Future acceptance of an artificial pancreas in adults with type 1 diabetes}

van Bon $A C^{1}$, Brouwer $T B^{2}$, von Basum $G^{3}$, Hoekstra JBL ${ }^{1}$, DeVries $J H^{1}$

${ }^{1}$ Department of Internal Medicine, Academic Medical Center Amsterdam, Amsterdam, The Netherlands; ${ }^{2}$ Department of Internal Medicine, Onze Lieve Vrouwe Gasthuis, Amsterdam, The Netherlands; and ${ }^{3}$ Philips Research, Eindhoven, The Netherlands

Diabetes Technol Ther 2011; 13: 731-736

\section{Background}

Patient acceptance of a closed-loop system is crucial to the future clinical implementation of a fully automated artificial pancreas system. Acceptance of individual AP components and acceptance of an AP in type 1 diabetes mellitus (T1DM) patients have been previously gauged using surveys and personal interviews, respectively. This study sought to develop a questionnaire to gauge future acceptance of an artificial pancreas and the factors influencing its acceptance in patients currently using conventional continuous subcutaneous insulin infusion (CSII) therapy.

\section{Methods}

Personal interviews with T1DM patients were conducted to explore the most important factors influencing $\mathrm{AP}$ use. Building off the results of the interviews, a questionnaire was developed using the Technology Acceptance Model (a survey model used to study acceptance of new computer systems utilizing the survey-taker's perceived usefulness, perceived ease of use, trust of, and intention to use the system as determinants of actual use). Before its administration to T1DM patients, an introduction describing the AP system was added, and the survey was analyzed to eliminate repetitive questions and verify question relevance. Numerous questions

\footnotetext{
${ }^{1}$ University of California at Santa Barbara, Santa Barbara, CA

${ }^{2}$ Sansum Diabetes Research Institute, Santa Barbara, CA

${ }^{3}$ Diabetes Technology Center, Jesse Z and Sara Lea Shafer Institute for Endocrinology and Diabetes, Schneider Children's Medical Center of Israel, Petah Tikva, Israel

${ }^{4}$ Sackler Faculty of Medicine, Tel Aviv University, Tel Aviv, Israel
} 
were omitted. The final questionnaire was administered to 200 patients.

\section{Results}

Of the questionnaires administered, 132 questionnaires were used. Most patients reported they would like to switch to the $\mathrm{AP}$ at the time of the questionnaire (intention to use). Most patients also believed that the AP would improve their glucose control and that it would reduce their frequency of hypo- and hyperglycemia (perceived usefulness). In addition, patients agreed that the AP seemed easy to use (perceived ease of use). Most patients were confident that the AP would deliver a correct amount of insulin (trust of system).

\section{Conclusions}

The results show that a majority of patients surveyed expressed a positive attitude toward the AP system and expected it to be beneficial. By gauging the perceived usefulness, perceived ease of use, patient trust of the $\mathrm{AP}$, and intention to use the $\mathrm{AP}$, the results of the questionnaire indicate urgency for more closedloop systems in type 1 diabetes. The questionnaire can be used to survey the future acceptance of the AP in other populations and to assess the potential acceptance of other features in the AP.

\section{Comment}

In the end, artificial pancreas systems are made for patients. While the benefits of the AP may be clear to healthcare providers and engineers, acceptance and adoption of the AP by patients is a key factor. Therefore, evaluating the acceptance of this technology and the patient's expectations is one of the important steps developers should consider. Some work has already been done in this area. This study in adults follows the study of Elleri et al. about parental attitudes toward overnight closedloop glucose control in children (1). As the authors state, the results may have been skewed by the fact that only about a third of the patients had previous experience with continuous glucose monitoring (CGM), which is an integral part of the AP system. The reliability of any questionnaire must also be taken into consideration, as results are susceptible to influence by patient lack of attention or lack of interest in the survey. Patients that had no previous knowledge of the AP may not have been able to properly visualize the AP system. It is important to mention that these patients are using pumps as was originally stated. Use of questionnaires should be an integral part of current AP clinical trials to give a preliminary evaluation of the benefit, usefulness, and comfort level of the participants.

\section{A controlled study of the effectiveness of an adaptive closed-loop algorithm to minimize corticosteroid- induced stress hyperglycemia in type 1 diabetes}

El Youssef $J^{1}$, Castle $J R^{1}$, Branigan $D^{2}{ }^{2}$, Massoud $R G^{2}$, Breen $M E^{1,2}$, Jacobs $P G^{3}$, Bequette $B W^{4}$, Ward $W K^{1,2}$

${ }^{1}$ Oregon Health \& Science University, Portland, Oregon; ${ }^{2}$ Legacy Health System, Portland, Oregon; ${ }^{3}$ Jacobs Technologies, Portland Oregon; and ${ }^{4}$ Rensselaer Polytechnic Institute, Troy, New York

J Diabetes Sci Technol 2011; 5: 1312-1326

\section{Background}

Changes in tissue insulin sensitivity can be caused by many factors, including illness, emotional stress, and treatment with certain medications. These changes pose a considerable challenge to closed-loop control. The purpose of this study was to examine the efficacy of a novel adaptive algorithm in responding to corticosteroid-induced changes in tissue insulin sensitivity.

\section{Methods}

An adaptive proportional derivative (APD) algorithm able to automatically adjust gain parameters was compared to a fading memory proportional-derivative (FMPD) algorithm, which uses fixed gain parameters in controlling glucose when insulin sensitivity was able to be lowered. Fourteen T1DM subjects were each studied for two 33-hour sessions: one of them utilizing the FMPD algorithm alone for the first 13 hours then switching to APD control for the last 20 hours, and the other consisting entirely of APD control. During both of the sessions, the patients were administered oral hydrocortisone starting with a loading dose of $40 \mathrm{mg}$ at hour three followed by six more doses of $20 \mathrm{mg}$ every four hours. Glucagon was administered subcutaneously to prevent hypoglycemia. Sessions began in the morning after overnight fasting, and the patients were given meals at 1, 5, 10, 25, and 29 hours after session commencement. Meal-announcement was used to deliver a premeal bolus consisting of $60 \%$ of the subject's normal premeal bolus. Subcutaneous (SC) glucose levels were measured using two Dexcom sensors, whose accuracy was measured by comparison with plasma glucose levels measured using the HemoCue 201 device. If the absolute relative difference (ARD) of the sensor being used exceeded $30 \%$ in the preceding $30 \mathrm{~min}$, the algorithm was switched to the other sensor. Insulin and glucagon rates were manually adjusted by a nurse every $5 \mathrm{~min}$ according to the algorithm's computations.

\section{Results}

In the session entirely under APD control, glucose increments three and four hours post-lunch (postprandial increase in glucose when compared to preprandial levels) were significantly smaller on day two than on day one, but not one and two hours post-meal. The APD did not significantly increase time spent in the hypoglycemic range when compared with FMPD. During the initial 13-hour "control" period, the APD algorithm maintained lower glycemia, on average 35$50 \mathrm{mg} / \mathrm{dL}$ less, than the FMPD algorithm. A four-hour delay in the effect of hydrocortisone was observed, with an average drop in insulin sensitivity of $40 \%$ to $50 \%$.

\section{Conclusions}

The APD algorithm appeared to maintain glycemic control better than the FMPD algorithm. Average glycemia during the initial 13-hour control period with APD was lower than the glycemic average for the FMPD period. Postprandial control following the lunch meal was used to determine if the APD algorithm effectively adapted to changing insulin requirements, since no dinner was given on day two and glycemia after breakfast on day one was still influenced by prestudy insulin dosages. The significantly smaller glucose increments on day two, when compared with day one, show 
that the APD algorithm was indeed adaptable. The use of glucagon was able to effectively prevent hypoglycemia.

\section{Comment}

The ability of a control algorithm to automatically adjust insulin delivery in response to changing insulin sensitivity due to stress is a valuable attribute in any closed-loop system. The need for higher basal insulin (> 70\% increase above nominal rates) in conditions of stress was demonstrated by Bevier et al. using similar protocol (2). While in this current study insulin-resistance was induced with orally administered steroids, further testing with other factors influencing insulin sensitivity, such as exercise, illness, and alcohol, could provide insight into the ability of this algorithm to handle such disturbances. The results are certainly interesting, but this study leaves some questions unanswered. No data for glucagon delivery is given, and a discussion on the advantages and disadvantages of glucagon and bihormonal control is absent. Postprandial glucose was surprisingly high on day two even with a $60 \%$ premeal bolus, which may suggest a conservative tuning on the control algorithm.

Two important questions remain unanswered regarding sensor use in future outpatient artificial pancreas studies. The first is whether SC sensors are accurate enough for use in the evaluation of study endpoints in outpatient closedloop studies. The second is how to increase the accuracy of the sensor signal that feeds into the AP system. In this study, the authors did not describe whether the outcomes were based on sensors' glucose levels or on reference HemoCue measurements. In addition, two sensors were used to increase the accuracy of the sensor signal. It is true that currently, the use of dual sensors provides a way to circumvent individual sensor inaccuracies (3), but this is not realistic in a commercial artificial pancreas. Certain recent developments in the development of a redundant CGM utilizing two sensing techniques that have different modes of failure might be able to provide enhanced and more reliable CGM signals for the AP. All of these concerns suggest a longer road to more elaborate, possibly outpatient-style clinical trials for this closed-loop design.

\section{Safety and efficacy of 24-h closed-loop insulin delivery} in pregnant women with well-controlled type 1 diabetes

Murphy $H R^{1}$, Kumareswaran $K^{1}$, Elleri $D^{1,2}$, Allen $J M^{1}$, Caldwell $K^{1}$, Biagioni $M^{1}$, Simmons $D^{3}$, Dunger $D B^{2}$, Nodale $M^{1}$, Wilinksa $M E^{1,2}$, Amiel $S A^{4}$, Hovorka $R^{1,2}$

${ }^{1}$ Metabolic Research Laboratories and the National Institute for Health Research Cambridge Biomedical Research Centre, University of Cambridge, Cambridge, UK; ${ }^{2}$ Department of Pediatrics, University of Cambridge, Cambridge, UK; ${ }^{3}$ Cambridge University Hospitals National Health Service Foundation Trust, Addenbrooke's Hospital, Cambridge, UK; and ${ }^{4}$ Kings College Hospital, Guy's, King's, and St. Thomas', London, UK

Diabetes Care 2011; 34: 2527-2529

\section{Background}

It is well established that hyperglycemia should be avoided in pregnancy, and that tight blood glucose control positively affects pregnancy outcomes. In previous studies, closed-loop insulin delivery systems were able to prevent nocturnal hypoglycemia and maintain BG levels within the target range $84 \%$ to $100 \%$ of the time in pregnant women with T1DM. The present study aimed to determine the efficacy and safety of 24-hour closed-loop insulin delivery in pregnancy, with exercise and meal disturbances.

\section{Methods}

Twelve pregnant women with type 1 diabetes in the United Kingdom participated in this randomized crossover trial of closed-loop insulin therapy and conventional continuous subcutaneous insulin infusion (CSII) therapy. Each woman participated in two 24-hour sessions, one of closed-loop insulin delivery and the other of conventional CSII, one to six weeks apart. During the sessions, venous blood samples were drawn every 15 to $30 \mathrm{~min}$ to obtain blood glucose reference values. At set times in each session, participants walked three times for $20 \mathrm{~min}$ and twice for $50 \mathrm{~min}$. Meals were consistent between sessions and snacks were given prior to exercise based on BG level. During closed-loop control, insulin delivery was adjusted every 15 min based on the CGM glucose levels and the control algorithm computations. During conventional CSII, the women set their own basal rates. During both sessions, the target BG range was 63 to $140 \mathrm{mg} / \mathrm{dl}$. Hypoglycemia was defined as less than or equal to $54 \mathrm{mg} / \mathrm{dl}$ with symptoms or $45 \mathrm{mg} / \mathrm{dl}$ without symptoms and was treated with $15 \mathrm{~g}$ oral carbohydrate. The primary outcome was time spent with plasma glucose levels in the target range. Secondary outcomes were time spent above and below target range, mean BG concentration, BG standard deviation, and low blood glucose index (LBGI). Outcomes of conventional CSII control and closed-loop control were compared and analyzed.

\section{Results}

For both closed-loop and conventional CSII arms, patients spent a comparable amount of time within the target range, $81 \%$ for both groups. During closed-loop therapy, there were 13 hypoglycemic episodes, and during conventional CSII there were 20 hypoglycemic episodes. The low blood glucose index (LBGI) was lower with closedloop control. During sleep, glucose levels were elevated but comparable between both visits, although less time was spent in the target range with conventional CSII than with closed-loop therapy. CGM measurements and plasma glucose measurements for time spent within target range were not consistent in the overnight period of conventional CSII treatment, with plasma measurements showing $98 \%(84-100)$ of time spent in target range and CGM showing $83 \%$ (50-100).

\section{Conclusions}

Closed-loop therapy was as effective as conventional CSII over a 24-hour period, with a lower risk of hypoglycemia. Closed-loop control did not prevent exercise-induced hypoglycemia, which is a challenge even dual hormone algorithms have not overcome. 


\section{Comment}

Tight glucose control during pregnancy is extremely important since it might have an imprint on the rest of the extrauterine life of the fetus $(4,5)$. This study attempted to emulate the real life of pregnant women by incorporating meals, exercise, and overnight periods, all of which provide challenges to good blood glucose management. However, there were some limitations to the study. The discrepancy in glucose measurements in the conventional CSII treatment period between CGM and plasma measurements required the study to rely on plasma glucose instead of CGM values. This underscores the need for more accurate CGMs to make closed-loop systems a realistic option for individual everyday use. The sample size was small, with only 12 subjects. Additionally, although the participants underwent periods of exercise, there is no explanation or analysis on the effects of this exercise on blood glucose levels. Few closed-loop studies have incorporated exercise into their protocol. More data are needed in this area to further develop appropriate closed-loop algorithms, especially in the challenging population of pregnant women.

\section{The identifiable virtual patient model: comparison of simulation and clinical closed-loop study results}

Kanderian $S S^{1}$, Weinzimer $S A^{2}$, and Steil $G M^{3}$

${ }^{1}$ Independent Consultant, Germantown, Maryland; ${ }^{2}$ Yale University, New Haven, Connecticut; and ${ }^{3}$ Children's Hospital Boston $\mathcal{E}$ Harvard Medical School, Boston, Massachusetts

J Diabetes Sci Technol 2012; 6: 371-379

\section{Background}

This study aimed to determine if the identifiable virtual patient (IVP) model could adequately emulate the results of a clinical study of closed-loop insulin delivery in pediatric patients.

\section{Methods}

Ten virtual patients were studied using the same protocol and procedures as in a previous clinical study of closed-loop insulin delivery in pediatric patients. In both studies, closedloop began at 7 a.m. and continued until 3 p.m. the next day. Three meals were given, at 8 a.m., 12 p.m., and 5 p.m. In the clinical study, eight patients were studied with full closed-loop (FCL) control (no manual meal boluses), and nine were studied with hybrid closed-loop (HCL) control (meal boluses $15 \mathrm{~min}$ prior to meal). In the simulator, all 10 virtual patients were studied with both FCL and HCL. In the case of hypoglycemia (below $60 \mathrm{mg} / \mathrm{dl}$ ), $15 \mathrm{~g}$ of carbohydrate were administered.

\section{Results}

The peak postprandial glucose levels between the two studies were comparable. During full closed-loop control, the postprandial median blood glucose for all meals in the virtual patients was $227 \mathrm{mg} / \mathrm{dl}$ (IQR 210-254) compared with $229 \mathrm{mg} / \mathrm{dl}$ (IQR 181-272) for the clinical pediatric patients.
The meal bolus in hybrid closed-loop significantly lowered the peak postprandial glucose compared to full closed-loop in both studies (median for simulated HCL, $206 \mathrm{mg} / \mathrm{dl}$ [IQR 184-228] and median for clinical HCL, $189 \mathrm{mg} / \mathrm{dl}$ [IQR 161225]). The time between peak insulin delivery and peak plasma insulin concentration for each meal was similar in both studies. However, approximately two thirds more insulin was delivered in the clinical study versus the simulation. Glucose remained in the target range $85 \%$ of the time in the clinical study, and $71 \%$ of the time in the simulation. There were three occurrences of hypoglycemia over 17 patient days in the clinical study versus seven occurrences over 20 patient days in the simulation, but the difference was not statistically significant.

\section{Conclusion}

Closed loop simulations of adult subjects using models like IVP are capable of reproducing the results of a clinical study of a pediatric population. Despite the differences in population, the simulation closely emulated the clinical study, with the exception that approximately one third less insulin was administered to the simulation subjects.

\section{Comment}

In silico simulations are a valuable tool in the development of the AP. Comparison studies of simulated and clinical trials are imperative in evaluating simulation algorithms. The UVA/Padova metabolic simulator (6) and others have enabled faster transitions from the bench to clinical studies. The authors recognize limitations to their comparison, such as slight meal differences between the two studies. Clinical patients consumed more carbohydrates at lunch than breakfast, while simulated meals had constant carbohydrate content for all three meals. The study was further limited by the different number of adults and children in each population. The authors also noted the slightly lower percentage of time spent in the target glucose range during the simulation (71\% simulation vs. $85 \%$ clinical), which they attributed to sensor bias in the clinical study resulting in underestimated glucose values. The results presented similar but not identical trends, and they suggest that the simulation results could be used to further improve the control algorithm. This study showed encouraging results, but the limitations indicate that further comparison studies should be performed.

\section{ARTIFICIAL PANCREAS IN THE ICU}

\section{Blood glucose control using an artificial pancreas reduces the workload of ICU nurses}

Mibu $K^{1}$, Yatabe $T^{2}$, Hanazaki $K^{3}$

${ }^{1}$ Nursing Department, Kochi Medical School Hospital, Kohasu Oko-cho, Nankoku, Kochi, Japan; ${ }^{2}$ Department of Anesthesiology and Critical Care Medicine, Kochi Medical School, Kohasu Oko-cho, Nankoku, Kochi, Japan; and ${ }^{3}$ Department of Surgery, Kochi Medical School, Nankoku, Japan

J Artif Organs 2012; 15: 71-76 


\section{Background}

Postoperative patients admitted to the ICU are likely to develop hyperglycemia as a result of the stress of surgery on the body. The prognosis of ICU postoperative patients worsens for patients with hyperglycemia, which may induce infections. Currently the sliding scale method of insulin dosing is used in the ICU, but with this method, target BG levels are only reached $22.7 \%$ of the time and are generally too high. Additionally, some incidents of uncontrolled BG can be attributed to mistakes in dosing by ICU nurses. This study aimed to determine whether the use of the STG-22 artificial pancreas is able to decrease the workload of ICU nurses and increase their awareness of BG monitoring in the ICU.

\section{Methods}

The study included 45 ICU patients split into two groups: the conventional management sliding scale method (SS) group and the artificial pancreas (AP) group. The SS method BG target was 150-200 mg/dl with BG levels being measured every four hours. Since the AP posed little risk of hypoglycemia, the target BG range was lower, at $80-110 \mathrm{mg} / \mathrm{dl}$. Measured quantities for both groups were (1) frequency of hypoglycemia (BG $<70 \mathrm{mg}$ / dl), (2) mean BG levels, and (3) amount of insulin administered. Workload of nurses was determined by measuring the time nurses spent working on glucose management, the number of BG readings taken, the number of double checks, and the number of calls made to the attending physician. For the SS group, time working on glucose management was defined as the time required to measure BG, confirm the insulin dosing protocol, and complete the insulin administration. For the AP group, time working on glucose management was defined as the time spent attending to alarms, due to disturbances such as an interruption of blood flow to the AP. To determine any change in awareness of BG monitoring due to the implementation of the AP, the nurses were given a three-question survey about BG awareness.

\section{Results}

There was no significant difference in the background factors of the patients between the two study groups. The amount of insulin administered per 24-hour period was significantly higher in the AP group than the SS group (136 \pm 135 vs. $25 \pm 25$ units). BG levels in the AP group were significantly lower than in the SS group (123 \pm 34 vs. $198 \pm 60 \mathrm{mg} / \mathrm{dl})$. Blood was sampled significantly less frequently in the AP group than the SS group (1.3 \pm 1.4 vs. $8.9 \pm 8.1$ times/admission), and the number of double checks was significantly less in the AP group than the SS group (1.0 \pm 1.4 vs. $9.8 \pm 8.5$ times/admission). The number of calls to the physician was significantly lower in the AP group than the SS group (0.6 \pm 1.1 vs. $2.2 \pm 1.8$ times/admission), and the time needed per admission was significantly shorter in the AP group. The burden of monitoring BG on the SS method vs. the AP method was much greater. From the survey results, the implementation of the AP allowed the nurses to become more aware of BG management and gave them a greater sense of security in managing BG.

\section{Conclusion}

The AP decreased the time nurses spent managing BG levels in ICU patients. Because the SS method is associated with the risk of hypoglycemia, and the AP had little risk of inducing hypoglycemia, the nurses admitted to having less anxiety while using the AP. The AP also increased the ICU nurses' awareness of BG monitoring, and even if a stricter target BG range is needed, their workload will not increase. The authors estimated that $\$ 14,000$ USD per year in personnel expenses could be saved if the AP is implemented in their hospital. The AP reduced the incidence of hypoglycemia because it eliminated the risk of human error in insulin dosing.

\section{Retrospective study on the effect of tight glucose control in postoperative sepsis patients using an artificial pancreas}

Takahashi $G^{1}$, Matsumoto $N^{1}$, Shozushima $T^{1}$, Onodera $C^{1}$, Kan $S^{1}$, Akitomi $S^{1}$, Hoshikawa $K^{1}$, Kikkawa $T^{1}$, Kojika $M^{1}$, Inoue $Y^{1}$, Suzuki $K^{2}$, Wakabayashi $G^{3}$, Endo $S^{1}$

${ }^{1}$ Department of Critical Care Medicine, Iwate Medical University, School of Medicine, 19-1 Uchimaru, Morioka, Japan; ${ }^{2}$ Department of Anesthesiology, Iwate Medical University, School of Medicine, Morioka, Japan; and ${ }^{3}$ Department of Surgery, Iwate Medical University, School of Medicine, Morioka, Japan

J Infect Chemother 2011; 17: 812-820

\section{Background}

Postoperative sepsis patients in the ICU require safe and reliable means of blood glucose control. An artificial pancreas has been used in prior studies in the ICU as an automated means of blood glucose measurement and insulin administration.

\section{Methods}

Twelve postoperative sepsis patients on mechanical ventilation in the ICU were selected for a seven-day study. The patients were divided into two groups of six, half receiving tight glucose control (TGC), and half receiving normal glucose control (GC). The TGC group was constantly monitored by the STG-22 artificial pancreas with a target blood glucose range of 80-150 mg/dl. The GC group had their glucose levels measured three times per day. Insulin dosages were determined on a sliding scale and were given by subcutaneous injection. Glucose was administered in the case of hypoglycemia (60 mg/ dl or less) $30 \mathrm{~min}$ after injection. Blood samples were taken every other day after surgery and tested for interferon (INF)- $\gamma$, interleukin (IL)-6, interleukin (IL)-10, and monocyte chemoattractant protein (MCP)-1. Blood C-peptide and insulin levels were evaluated to quantify endogenous insulin production. Peripheral blood was assayed to determine the level of high mobility group box protein (HMGB)-1 and soluble receptor for advanced glycation end products (sRAGE). These parameters were measured to gauge the inflammatory status of each patient pre- and posttreatment. Changes in all of the measured variables were analyzed by comparing each with the baseline levels of each patient taken prior to starting the study.

\section{Results}

The mean blood glucose (BG) level for the TGC group was $129.7 \pm 9.7 \mathrm{mg} / \mathrm{dl}$, and for the GC group it was $200.0 \pm 14.7 \mathrm{mg} /$ $\mathrm{dl}$, and BG levels were controlled within the target range for the TGC group. The insulin dosages were much larger for the TGC 
group as compared with the GC group. The serum C-reactive protein (CRP) decreased steadily in the TGC group, while in the GC group CRP peaked on day three at $17.5 \mathrm{mg} / \mathrm{dl}$. Serum INF- $\gamma$ showed no significant changes over time or between groups. IL-6 decreased comparably in both groups, while serum IL-10 remained unchanged in the TGC group but tended to decrease in the GC group, although the difference between the two groups was insignificant. HMGB-1 tended to decrease in the TGC group, but there was no significant intergroup difference. Serum sRAGE rose from day three in the TGC group but there was no significant intergroup difference. Serum MCP-1 remained lower in the TGC group than in the GC group throughout the sevenday study period. There were no significant differences between the two groups in any of these parameters in the baseline levels.

\section{Conclusions}

According to these results, the artificial pancreas can be considered safe for use on sepsis patients in the ICU, as no instances of hypoglycemia occurred. These results suggest that changes in IL-10, MCP1, s100A12, HMGB-1, and sRAGE may be useful for evaluation of impact on glucose control in sepsis patients. However, because serum levels of cytokines such as INF- $\gamma$, IL-6, and CRP are known to rise in the presence of infectious and noninfectious stresses, their measurement does not allow for decisive conclusions to be drawn about the effects of tight glucose control in sepsis. Additionally, the decrease in C-peptide in the TGC group is a result of the higher levels of exogenous insulin administered by the AP. Because the device was successful in keeping the patients within the target BG range and avoiding hypoglycemia, further studies with more patients are indicated.

\section{Comment}

Tight glucose control in the ICU is still a controversial topic. However, the ICU seems like a natural place for implementation of the artificial pancreas. The artificial pancreas is a safe and useful method of monitoring BG in ICU patients, and should be considered as a tool to manage BG in a perioperative setting. These limited-size studies elucidated some of the advantages to implementation of the AP in the ICU. For example, the AP reduced the time and stress of the nurses responsible for checking blood glucose and may become a cost-cutting strategy for hospitals. However, as the authors state, the different target blood glucose levels for the AP and SS groups may compromise the integrity of the studies. One drawback of AP used in that study is that it requires a large amount of blood, possibly leading to anemia. Additionally, the interrupted blood flow to the AP may be dangerous to the patient and ultimately cause more work for the nurses. Improved technology, possibly by using a central vein instead of a peripheral vein, could eliminate interruptions in blood flow and make the AP even more suitable for use in the ICU. These studies took a step toward implementation of the artificial pancreas and showed that the AP could be successfully used in patients other than type 1 diabetics. Multicenter, multinational studies with suitable number of patients are needed to demonstrate the applicability of introducing the artificial pancreas into ICU units in the world.

\section{UNDER THE HOOD}

\section{Closed-loop insulin delivery utilizing pole placement to compensate for delays in subcutaneous insulin delivery}

\author{
Loutseiko $M^{1}$, Voskanyan $G^{1}$, Keenan $D B^{1}$, Steil $G M^{2}$ \\ ${ }^{1}$ Medtronic MiniMed, Northridge, California; and ${ }^{2}$ Children's \\ Hospital Boston, Boston, Massachusetts
}

J Diabetes Sci Technol 2011; 5: 1342-1351

\section{Background}

Some of the hurdles in the design of closed-loop AP are the delays associated with the subcutaneous (SC) pharmacokinetic (PK)/pharmacodynamic (PD) profile and those associated with SC glucose physiology. An insulin feedback (IFB) term in the control algorithm can help in preventing insulin over delivery. This article investigates the addition of an IFB component to a proportional-integral-derivative (PID) control algorithm currently under construction for closed-loop control.

\section{Methods}

Closed-loop AP evaluation was done on diabetic dogs using CSII and the Medtronic PID algorithm with IFB. Blood glucose measurements were taken intravenously every 10-20 min and immediately analyzed. The BG data were subjected to the same digital filters as regular CGM data to recreate any signal delay from the filters. Plasma from each time-point was stored for later analysis of plasma insulin concentrations and comparison with predicted insulin concentration.

\section{Results}

Increasing levels of IFB gain were correlated with lower peak postprandial glucose levels and lower glucose area under the curve during the closed-loop period. Total insulin delivered was not different with different levels of IFB gain, although with higher IFB gain more insulin delivery occurred earlier in the meal response. The predicted plasma concentrations of insulin were well correlated with measured plasma insulin concentrations.

\section{Conclusions}

Extending the PID algorithm to include an IFB gain component was shown to improve the closed-loop meal response in diabetic dogs. Feedback loops for other components of the AP algorithm could improve the meal response even further, although optimally configuring a larger number of control parameters could require the use of a computer-simulation model.

\section{Comment}

The IFB component of the PID algorithm described in this study shows promise for improving the meal response in a closed-loop AP situation, which remains a large challenge in any AP design. As noted by the authors, canine glycemia may differ from human glycemia in subtle but significant ways that may impede the direct application of this algorithm to human physiology, even though the 
results suggest that they are comparable. It should also be noted that while the data in this study are promising, no CGM was utilized. The intravenous BG data were subjected to the same delays as CGM data, but their accuracy was not modified to reflect physiological differences between interstitial and intravenous glucose levels. An interstitial CGM should be used instead of plasma glucose in future modification and assessment of the IFB component in overcoming meal challenges with the current available glucose sensors without late postprandial hypoglycemia both in diabetic dogs as well as in human trials.

\section{MPC-based artificial pancreas: strategies for individualization and meal compensation}

Soru $P^{1}$, De Nicolao $G^{1}$, Toffanin $C^{1}$, Dalla Man $C^{2}$, Cobelli $C^{2}$, Magni $L^{3}$

${ }^{1}$ Department of Industrial and Information Engineering, University of Pavia, Pavia, Italy; ${ }^{2}$ Department of Information Engineering, University of Padova, Padova, Italy; and ${ }^{3}$ Department of Civil Engineering and Architecture, University of Pavia, Pavia, Italy

Annual Reviews in Control 2012; 36: 118-128

\section{Background}

Model-predictive control (MPC) algorithms have shown encouraging results for use in a closed-loop artificial pancreas system. This article describes a linear-MPC structure that can incorporate knowledge from conventional insulin therapy into a closed-loop AP.

\section{Methods}

This article compared three control algorithms and their performance in silico in a cohort of 100 virtual patients: I-MPC, or ideal MPC, assumed everything about the patient was known (this strategy is only feasible for virtual patients); MPC1, or average model design, which used average patient data obtained from the in silico patients; and MPC2, or individualized model design, which used an individualized controller assuming only certain individual patient parameters were known, such as the carb-ratio and average daily basal insulin amount. Each of the algorithms were tested in simulation in four scenarios: 1) a nominal scenario consisting of an $80 \mathrm{~g}$ dinner, a $50 \mathrm{~g}$ breakfast, and a $60 \mathrm{~g}$ lunch; 2) the nominal scenario in which the amount of carbohydrates ingested is multiplied by a random factor ranging between 0.5 and 1.5, meant to simulate error in meal carbohydrate content; 3 ) the nominal scenario with a $\pm 25 \%$ change in insulin sensitivity, meant to represent uncertainty in insulin sensitivity; and 4) the nominal scenario with a $25 \%$ increase in nominal basal insulin, meant to simulate inaccurate open-loop therapy.

\section{Results}

Across all scenarios, the I-MPC performed well, with mean glucose levels always within the target range $(70-180 \mathrm{mg} / \mathrm{dl})$, except after meals, and average overall time spent in target range from $96 \%-99 \%$ across the four scenarios. The average model design, MPC1, showed mean glucose levels generally higher after meals and overall average time in target range between $86 \%-94 \%$ across the 4 scenarios. The individualized model, MPC2, showed average glucose levels similar to those of the I-MPC, with an average time in target range of between $94 \%-98 \%$. With regard to time spent in tight target range $(80-140 \mathrm{mg} / \mathrm{dl})$, the I-MPC performed the best $(86 \%-$ $99 \%$ across the four scenarios), the MPC2 almost as well ( $84 \%-$ $98 \%)$, and the MPC1 slightly worse (71\%-94\%).

\section{Conclusions}

This article describes the development and evaluation of several MPC algorithms that exhibited good performance in silico in comparison with the algorithm identified by the authors as ideal but not implementable (I-MPC). Both implementable algorithms exhibited good performance, with the individualized model design MPC2 proving slightly advantageous to the average model design MPC1. The average model design MPC1 was recently used in a multicenter study along with a safety supervision module, with results expected to be available in the coming months. However, since this study shows that the individualized model design MPC2 may offer improved performance over MPC1, further investigation is needed to determine how to incorporate more elaborate individualization into AP control algorithms.

\section{Comment}

These results are encouraging and certainly merit further inspection. Both individualization of the controller based on available clinical parameters and obtaining a personalized patient model have been explored in various in silico, as well as clinical, studies, and both are valid approaches. The current design relies on a linear unconstrained MPC to minimize computational power, even though computational power is not a limiting factor for MPC. This limits the ability of MPC to optimally handle constraints. The use of meal announcement/manual meal delivery is an integral part of the design and may limit the ability of the controller to regulate unannounced meals. The two designs should be further evaluated in a clinical setting and compared with an open-loop strategy (basal bolus), since this is the basis of the suggested design.

\section{A bio-inspired glucose controller based on pancreatic $\beta$-cell physiology}

Herrero $P^{1}$, Georgiou $P^{1}$, Oliver $N^{1,2}$, Johnston $D G^{1,3}$, Toumazou $C^{1}$

${ }^{1}$ Center for Bio-Inspired Technology, Institute of Biomedical Engineering, Imperial College London, London, United Kingdom; ${ }^{2}$ Charing Cross Hospital, Imperial College Healthcare NHS Trust, London, United Kingdom; and ${ }^{3}$ Mary's Hospital, Imperial College Healthcare NHS Trust, London, United Kingdom

J Diabetes Sci Technol 2012; 6: 606-616

\section{Background}

Most closed-loop artificial pancreas control algorithms utilize classical control techniques like PID and MPC, which are not based on normal physiology. The human body is a fascinating creation with multiple control loops. Bio-inspired technologies seeking to mimic normal pancreatic physiology 
may be more effective in the maintenance of euglycemia. This article introduced a novel glucose controller based on normal $\beta$-cell physiology and evaluated it in type 1 diabetes mellitus (T1DM) virtual populations.

\section{Methods}

The bio-inspired controller was evaluated in a subcutaneoussubcutaneous closed-loop design using the UVA/Padova metabolic simulator in adult and adolescent populations.

\section{Results}

Hypoglycemia was minimal in both the adult and adolescent populations; $6 \%$ of adults experienced BGs $<70$ for a mean $0.4 \%$ of the study duration, while $21 \%$ of adolescents experienced hypoglycemia for a mean $1.7 \%$ of study time. $2.0 \%$ of Adolescents experienced extreme hypoglycemia $(\mathrm{BG}<50)$ for a mean time of $0.1 \%$. The control-variability grid analysis (CVGA) graph for adolescents showed $67 \pm 17 \%$ of time spent in zones $\mathrm{A}$ and $\mathrm{B}$, and $1.3 \pm 3.1 \%$ of time spent in zones $\mathrm{D}$ and $\mathrm{E}$. The adolescent population showed a mean time spent in the hyperglycemic zone $(>180 \mathrm{mg} / \mathrm{dl})$ of $14.73 \pm 12 \%$, which corresponds to a high blood glucose risk index (HBGI) of $3.3 \pm 2.5$. Adult patients spent a mean time in the hyperglycemic range of $6.7 \pm 7.0 \%$ with $0.1 \pm 0.5 \%$ spent in the extreme hyperglycemic zone $(>300 \mathrm{mg} / \mathrm{dl})$ corresponding to an HBGI of $1.8 \pm 1.6$.

\section{Conclusion}

The bio-inspired glucose controller model has been shown to effectively control BG with minimal hyper- and hypoglycemia. Clinical trials are planned to validate the performance of this controller in real T1DM patients.

\section{Comment}

An artificial pancreas controller modeled after normal $\beta$ cell physiology provides an interesting alternative to current $\mathrm{AP}$ designs based on PID, FL and MPC controllers. Like many other control algorithms, bio-inspired control still must accommodate varying insulin sensitivities and other BG-influencing factors before it can be implemented in a clinical situation. Due to its bio-mimetic premise, this controller may be appropriate for use with a more physiological route of insulin administration, i.e. intraperitoneal delivery. This algorithm also utilizes meal announcement, a common closed-loop strategy to minimize the postprandial glycemic excursion. Meal-announcement strategies are able to improve post-prandial glycemia, but they also introduce a greater risk when meals are missed or are different from what was announced. Fully automated, non-announced meal compensation strategies, such as the one used in this study, offer a possible means of avoiding that risk, albeit at the cost of higher postprandial glycemia.

\section{Artificial neural networks for closed-loop control of in silico and ad hoc type 1 diabetes}

Fernandez de Canete ${ }^{1}$, Gonzalez-Perez $S^{1}$, Ramos-Diaz JC ${ }^{2}$

${ }^{1}$ Department of System Engineering and Automation, Engineering School C/Dr. Ortiz Ramos s/n, Malaga, Spain; and ${ }^{2}$ Pediatric
Service, Antequera District Hospital, C/Poeta Munoz Rojas s/n, 29200 Malaga, Spain

Comput Methods Programs Biomed 2012; 106: 55-66

\section{Background}

Interpatient variations in insulin-glucose dynamics underline the need for an individualized approach to closedloop glycemic control. In this article, artificial neural networks (ANN) were used to create $a d$ hoc and in silico glucose-insulin models and ad hoc controllers using clinical data for a number of individual diabetic patients.

\section{Methods}

An artificial neural network of the insulin-glucose dynamics of each patient was developed using data obtained from the use of a subcutaneous (SC) glucose sensor, an insulin pump, and a portable meal calculator over a 30-day training period. This data was used with a learning algorithm to design an ad hoc model for each patient. In order to make the training data as broad as possible, each patient underwent periods of fasting, exercise, and excessive insulin bolus. The ad hoc insulin-glucose model was designed to take the measured parameters from the training period (quantity of SC insulin administered, measured SC glucose, food ingested, and amount of physical exercise) as inputs and produce a predicted SC glucose level as the output. A 5-min delay in sensor data and a 20-min delay in insulin absorption were incorporated into the model. The ANN model was validated through comparison with additional patient data that was not used in the development of the model. A corresponding control algorithm for each ad hoc, in silico model was also developed using ANN. Models and corresponding control algorithms were developed and tested in silico for 20 type 1 diabetic patients for simulation periods of 700 minutes, with five meals and four periods of physical activity.

\section{Results}

The average daily glucose, minimum glucose, and maximum glucose were $139.31 \pm 2.54 \mathrm{mg} / \mathrm{dl}, 76.16 \pm 3.36 \mathrm{mg} / \mathrm{dl}$, and $202.30 \pm 6.03 \mathrm{mg} / \mathrm{dl}$ respectively. The average percentage of time in the desired range, $70-180 \mathrm{mg} / \mathrm{dl}$, was $95.16 \pm 1.03 \%$. The average percentage of time spent hyperglycemic was $4.83 \pm 1.03 \%$. No patients reached the hypoglycemic range.

\section{Conclusions}

The in silico models showed good control for each ad hoc simulation and controller, with average root mean squared (RMS) and relative RMS values of $5.96 \pm 2.05 \mathrm{mg} / \mathrm{dl}$ and $4.08 \pm 1.27 \%$ respectively. Future clinical testing could provide further insight into the appropriateness of the ad hoc ANN approach to closed-loop control.

\section{Comment}

Neural net models and control algorithms can be found in different engineering applications; the use of NN for modeling and control of an AP is being explored by several groups around the world. The ability of the NN to 
learn and adapt is an important property that is useful to AP development but one that can also be achieved by other means. The construction of this algorithm required a large set of patient data that was collected during a period of one month (carbohydrate ingestion, insulin administration, and daily patterns in glucose profile), which raises questions around the feasibility of this method. Moreover, the design and computation intensive nature of ANNs also brings into question the realistic applicability of this approach. A commercial AP device must be able to be applied easily and efficiently to large numbers of patients, optimally in the form of a handheld device, and this may not be possible using ANN algorithms despite the claims of the authors. The presented results are impressive, but it is unclear if they can be reproduced on a real subject or using a different simulation environment.

\section{An insulin infusion advisory system based on autotuning nonlinear model-predictive control}

Zarkogianni $K^{1}$, Vazeou $A^{2}$, Mougiakakou $S G^{3,4}$, Prountzou $A^{1}$, Nikita $K S^{5}$

${ }^{1}$ Biomedical Simulations and Imaging Laboratory, National Technical University of Athens, Athens, Greece; ${ }^{2}$ Diabetes Center, First Department of Pediatrics, PEA Kyriakou Children's Hospital, Athens, Greece; ${ }^{3}$ Institute of Communication and Computer Systems, National Technical University of Athens, Athens, Greece; ${ }^{4}$ Faculty of Medicine, Artificial Organ Center for Biomedical Engineering Research, and the University Hospital-InselspitalDivision of Endocrinology, Diabetes and Clinical Nutrition, University of Bern, Bern, Switzerland; and ${ }^{5}$ Faculty of Electrical and Computer Engineering, National Technical University of Athens, Zografou, Athens, Greece

IEEE Transactions on Biomedical Engineering 2011; 58: 2467-2477

\section{Background}

The most common approaches to closed-loop BG control are based on proportional derivative control or MPC (linear and nonlinear) algorithms. MPC seems most suitable for the $\mathrm{AP}$ because it can handle problems related to the nonlinearity of glucose-insulin metabolism, time delays in SC insulin absorption, time delays in food absorption, and noise in the SC glucose measurements. Attempts have been made to evaluate linear MPC and nonlinear MPC (NMPC). Clinical trials on real patients using NMPC have been performed to investigate the possibility of overnight BG control with NMPC. One challenge is tuning the MPC for each individual, but recently an online adaptive strategy enabling automatic tuning of parameters has been proposed.

\section{Methods}

This study aimed to design, develop, and evaluate a novel insulin infusion advisory system (IIAS) that uses a personalized model for glucose-insulin regulation in T1DM patients. The proposed IIAS includes two models. The first is the personalized glucose-insulin metabolism model, which provides the controller with glucose predictions ahead of time. The second is the NMPC, which provides estimates of the future outputs of the system. Additionally, the algorithm contains the automatic online tuning algorithm. The IIAS was tested in silico using the UVA/Padova T1DM simulator on the 10 adult patients available in the training version of the simulator. The IIAS was evaluated based on the predictive performance of the personalized model and ability to control BG in different simulated scenarios.

\section{Results}

In comparison with the fixed parameter NMPC over 48 hours, the NMPC resulted in two hypoglycemic episodes (BG $<60 \mathrm{mg} / \mathrm{dl}$ ), while the IIAS maintained target BG levels throughout the study. The control variability grid analysis (CVGA) indicated that IIAS controlled BG concentration better than NMPC. IIAS kept BG within the target range $95.17 \%$ of the time for all 10 patients, and the risk associated with extreme BG variations was low, indicating tight glycemic control. IIAS was able to handle meal estimation errors without severe hypo- or hyperglycemic episodes, in addition to intraday variation in physiological parameters. IIAS was also compared to adaptive basal therapy and was found to maintain tighter glucose control.

\section{Conclusion}

The novel IIAS was found to be a reliable system of closedloop glucose monitoring in this in silico study. IIAS can manage sensor error, lags, meal disturbances, large meal estimation errors, interpatient variability, and intraday variation in physiological parameters.

\section{Comment}

Developing the most suitable algorithm for an artificial pancreas has been the focus of much research. This complex approach was developed based on simulated data (several days long) with no day-to-day variability. The use of a nonlinear approach in the development of an $\mathrm{AP}$ is yet to be justified since linear methods have proven to be sufficient. The use of this method may be problematic with a wearable AP due to the large computational requirement. Nevertheless, the in silico results are promising. While in silico studies provide a basis for the efficacy of a particular BG algorithm, real-patient clinical studies are needed to determine if the IIAS algorithm is able to control blood glucose as well for real patients.

\section{Automatic learning algorithm for the MD-Logic artificial pancreas system}

Miller $S^{1}$, Nimri $R^{1}$, Atlas $E^{1}$, Grunberg $E A^{1}$, Phillip $M^{1,2}$

${ }^{1}$ The Jesse Z. and Sara Lea Shafer Institute for Endocrinology and Diabetes, The National Center for Childhood Diabetes, Schneider Children's Medical Center of Israel, Petah Tikva, Israel; and ${ }^{2}$ Sackler Faculty of Medicine, Tel-Aviv University, Tel-Aviv, Israel

Diabetes Technol Ther 2011; 13: 983-990

\section{Background}

One of the major issues facing closed-loop insulin delivery is the interpatient variability in insulin-glucose dynamics. 
This article presents the development and in silico evaluation of a learning algorithm for a previously established MD-Logic AP (MDLAP) system.

\section{Methods}

A learning algorithm designed to adjust the controller's correction index (CCI) was incorporated into the MDLAP system. The algorithm consists of an initial learning algorithm (ILA) component that takes data from conventional therapy periods to calculate an initial CCI, and a runtime learning algorithm (RLA) component that monitors closed-loop control and adjusts the CCI as necessary. The algorithm was tested in populations of adults, adolescents, and children using the University of Virginia/Padova simulator in seven different experiments designed to evaluate the ability of the algorithm components to appropriately adjust the CCI under normal conditions, conditions of altered insulin sensitivity, and conditions of artificially modified initial CCIs.

\section{Results}

The percentage of time spent with a BG between 70$180 \mathrm{mg} / \mathrm{dl}$ (per $\left.\mathrm{BG}_{70-180}\right)$ was significantly longer and time spent hypoglycemic was significantly lower for children and adolescents after activating both ILA and RLA when compared with control without ILA and RLA, but both of these measures were unchanged in the adult group. The per $\mathrm{BG}_{70-180}$ also increased for all groups with more time spent under ILA and RLA control, although time spent hypoglycemic did not change with more time under ILA and RLA control for any of the groups. A seven-fold reduction in the time spent in the hypoglycemic zone was observed following the use of the learning algorithm compared to the control group.

\section{Conclusions}

The learning algorithm developed in this study has the ability to learn and adapt to a patient's individual insulin needs and can be incorporated into any closed-loop algorithm that utilizes patient-specific parameters. The algorithm showed either improvement in glycemic control or maintenance of current glycemic control in a variety of simulated conditions. These results merit the future use of this learning algorithm in clinical trials to further validate its performance.

\section{Comment}

While promising, the results of any in silico study must be interpreted carefully due to the incomplete nature of the models that are used for simulation. Of note are the attempts to challenge the algorithm with random changes in meal estimation and insulin sensitivity. However, it would be of interest to evaluate the algorithm with both diurnal and dayto-day changes in insulin sensitivity and to evaluate the rate of convergence of the algorithm. Clinical evaluation of the algorithm is most likely the next logical step.

\section{Summary}

This past year has seen numerous advances in the artificial pancreas field. Simulators continue to facilitate the transition from the drawing board to clinical trials. By serving as a substitute for animal studies, in silico simulations have enabled the preliminary evaluation and approval of new control algorithms. Although proportional-integral-derivative (PID), model predictive control (MPC), and fuzzy logic (FL) control algorithms remain the most common control techniques for the $\mathrm{AP}$, there is increased interest in alternative approaches. Some of these nonmainstream algorithms have been highlighted in this chapter.

More and more the field is starting to recognize the importance of comparing in silico studies and in vivo clinical evaluation of algorithms. Simulation studies undeniably play an important role but it must be noted that in vivo and in silico performance can be vastly different. It is impossible to design a clinically applicable AP entirely in silico, since the simulators themselves are fundamentally incomplete. A large challenge still facing the closed-loop project is addressing the large intra- and interpatient variations in glucose-insulin dynamics (7) and the adaptation of simulators to better model these variations. Nonetheless, this year has seen more strides toward the individualization of controllers better able to accommodate such differences.

Some in the field have gone so far to say that the limitations of current CGMs are the largest challenge to AP closed-loop control. In addition to their inherent time lags, the main drawback to current CGMs is the possibility of accuracy drift and sensor failure $(8,9)$. With initial ambulatory studies now being undertaken, study designs with primary endpoints based on CGM values seem to be realistic. Current sensor accuracy not only affects the safety of the closed-loop system but also raises questions about the reliability of the evaluation of the AP system in outpatient settings. Some advances have been made in accommodating for the drawbacks of the SC route through the use of algorithm modifications. As Hovorka (8) and Zisser (9) note, up-and-coming technologies, such as fluorescence-based glucose sensing, have great potential in addressing some of the disadvantages of not only SC glucosesensing but also of SC insulin administration. Most of these new technologies are still quite immature; therefore, their incorporation into the AP will take time. In order to maintain the present momentum, other solutions should be found until these new technologies can be incorporated.

Safety of the AP is still of concern. Utilizing a modular approach to controller design, as described by Cobelli et al. (7), it is possible to conceive of independent development of safety algorithms for various components of the AP. In a commercial AP device, the system must be able to independently detect malfunctions such as catheter occlusion, malabsorption of insulin, and loss of sensor accuracy. There has been some interest in this area, but, like in all others, progress is gradual.

The AP field is starting to set realistic goals, such as focusing first on nocturnal closed-loop control, helping to move the project forward slowly but surely. The development of a closed-loop artificial pancreas system may be slow, but with initial ambulatory studies now being undertaken and a significant push toward commercialization, progress is certainly being made.

\section{Author Disclosure Statement}

E.D., T.H., J.F., and E.A. declare that no competing financial interests exist. M.P. is a member of advisory boards for AstraZeneca, Sanofi, Medtronic, and Eli Lily. He is a consultant to Bristol Myers-Squibb, AstraZeneca, and Andromeda. He is 
on the speaker's bureau of Johnson and Johnson, Sanofi, Medtronic, Novo Nordisk, and Roche. He is a shareholder of CGM-3 and D-Medical.

\section{References}

1. Elleri, D, Acerini, CL, Allen, JM, Hayes, J, Pesterfield, C, Wilinska, ME, Dunger, DB, Hovorka, R. Parental attitudes towards overnight closed-loop glucose control in children with type 1 diabetes. Diabetes Technol Ther 2010; 12: 35-9.

2. Bevier, WC, Zisser, HC, Jovanovič, L, Finan, DA, Palerm, CC, Seborg, DE, Doyle III, FJ. Use of continuous glucose monitoring to estimate insulin requirements in patients with type 1 diabetes mellitus during a short course of prednisone. J Diabetes Sci Technol 2008; 2: 578-83.

3. Castle, JR, and Ward, WK. Amperometric glucose sensors: sources of error and potential benefit of redundancy. J Diabetes Sci Technol 2010; 4: 221-5.
4. Jovanovič, L, Peterson, CM, Saxena, BB, Dawood, MY, Saudek, CD. Feasibility of maintaining normal glucose profiles in insulin-dependent pregnant diabetic women. Am J Med 1980; 68: 105-12.

5. Jovanovič, L, Druzin, M, and Peterson, CM. Effect of euglycemia on the outcome of pregnancy in insulin-dependent diabetic women as compared with normal control subjects. Am J Med 1981; 71: 921-7.

6. Kovatchev, BP, Breton, M, Dalla Man, C, Cobelli, C. In silico preclinical trials: a proof of concept in closed-loop control of type 1 diabetes. J Diabetes Sci Technol 2009; 3: 44-55.

7. Cobelli, C, Renard, E, and Kovatchev, B. Artificial pancreas: past, present, future. Diabetes 2011; 60: 2672-82.

8. Hovorka, R. Closed-loop insulin delivery: from bench to clinical practice. Nat Rev Endocrinol 2011; 7: 385-95.

9. Zisser, H. Clinical hurdles and possible solutions in the implementation of closed-loop control in type 1 diabetes mellitus. J Diabetes Sci Technol 2011; 5: 1283-6. 